PROCEEDINGS OF THE

AMERICAN MATHEMATICAL SOCIETY

Volume 133, Number 3, Pages 655-660

S 0002-9939(04)07728-7

Article electronically published on October 7, 2004

\title{
ASSOCIATED PRIMES OF LOCAL COHOMOLOGY MODULES
}

\author{
KAMRAN DIVAANI-AAZAR AND AMIR MAFI
}

(Communicated by Bernd Ulrich)

\begin{abstract}
Let $\mathfrak{a}$ be an ideal of a commutative Noetherian ring $R$ and $M$ a finitely generated $R$-module. Let $t$ be a natural integer. It is shown that there is a finite subset $X$ of Spec $R$, such that $\operatorname{Ass}_{R}\left(H_{\mathfrak{a}}^{t}(M)\right)$ is contained in $X$ union with the union of the sets $\operatorname{Ass}_{R}\left(\operatorname{Ext}_{R}^{j}\left(R / \mathfrak{a}, H_{\mathfrak{a}}^{i}(M)\right)\right)$, where $0 \leq i<t$ and $0 \leq j \leq t^{2}+1$. As an immediate consequence, we deduce that the first non-a-cofinite local cohomology module of $M$ with respect to $\mathfrak{a}$ has only finitely many associated prime ideals.
\end{abstract}

\section{INTRODUCTION}

Throughout this paper, $R$ is a commutative Noetherian ring with identity. For an ideal $\mathfrak{a}$ of $R$ and an $R$-module $M$, the $i$-th local cohomology module of $M$ with respect to $\mathfrak{a}$ is defined as

$$
H_{\mathfrak{a}}^{i}(M)=\underset{n}{\lim } \operatorname{Ext}_{R}^{i}\left(R / \mathfrak{a}^{n}, M\right)
$$

The reader can refer to 3 for the basic properties of local cohomology.

In [6], Hartshorne defines an $R$-module $M$ to be $\mathfrak{a}$-cofinite if $\operatorname{Supp}_{R} M \subseteq \mathrm{V}(\mathfrak{a})$ and $\operatorname{Ext}_{R}^{i}(R / \mathfrak{a}, M)$ is finitely generated for all $i \geq 0$. He asks when the local cohomology modules of a finitely generated module are $\mathfrak{a}$-cofinite. In this regard, the best known result is that for a finitely generated $R$-module $M$ if either $\mathfrak{a}$ is principal or $R$ is local and $\operatorname{dim} R / \mathfrak{a}=1$, then the modules $H_{\mathfrak{a}}^{i}(M)$ are $\mathfrak{a}$-cofinite. These results are proved in [8. Theorem 1] and [14, Theorem 1.1], respectively.

Since for an a-cofinite module $N$, we have $\operatorname{Ass}_{R} N=\operatorname{Ass}_{R}\left(\operatorname{Hom}_{R}(R / \mathfrak{a}, N)\right)$, it turns out that $\operatorname{Ass}_{R} N$ is finite. Huneke [7] raised the following question: If $M$ is a finitely generated $R$-module, then the set of associated primes of $H_{\mathfrak{a}}^{i}(M)$ is finite for all ideals $\mathfrak{a}$ of $R$ and all $i \geq 0$. Singh [12] gives a counterexample to this conjecture. However, it is known that this conjecture is true in many situations. For example, Brodmann and Lashgari [2, Theorem 2.2] showed that, if for a finitely generated $R$-module $M$ and an integer $t$, the local cohomology modules $H_{\mathfrak{a}}^{0}(M), H_{\mathfrak{a}}^{1}(M), \ldots, H_{\mathfrak{a}}^{t-1}(M)$ are all finitely generated, then $\operatorname{Ass}_{R}\left(H_{\mathfrak{a}}^{t}(M)\right)$ is finite. For a survey of recent developments on finiteness properties of local cohomology, see Lyubeznik's interesting article [10].

Received by the editors October 16, 2003.

2000 Mathematics Subject Classification. Primary 13D45, 13E99.

Key words and phrases. Local cohomology, associated prime ideals, cofiniteness, weakly Laskerian modules, spectral sequences. 
In this article, we first introduce the class of weakly Laskerian modules. This class includes all Noetherian modules and also all Artinian modules. Moreover, this class is large enough to contain all Matlis reflexive modules as well as all linear compact modules. Then as the main result of this paper, we establish the following. Let $M$ be a weakly Laskerian module and $t \in \mathbb{N}$ a given integer. There is a finite subset $X$ of Spec $R$ such that

$$
\operatorname{Ass}_{R}\left(H_{\mathfrak{a}}^{t}(M)\right) \subseteq\left(\bigcup_{0 \leq i<t, 0 \leq j \leq t^{2}+1} \operatorname{Ass}_{R}\left(\operatorname{Ext}_{R}^{j}\left(R / \mathfrak{a}, H_{\mathfrak{a}}^{i}(M)\right)\right)\right) \cup X .
$$

Clearly this result implies the main result of $[2]$.

\section{THE RESUlTS}

An $R$-module $M$ is said to be Laskerian if any submodule of $M$ is an intersection of a finite number of primary submodules. Obviously, any Noetherian module is Laskerian. Next, we present the following definition.

Definition 2.1. An $R$-module $M$ is said to be weakly Laskerian if the set of associated primes of any quotient module of $M$ is finite.

Example 2.2. i) Any Laskerian module is weakly Laskerian. In particular, any Noetherian module is weakly Laskerian.

ii) It is known that the set of associated primes of an Artinian module is a finite set consisting of maximal ideals. Hence any Artinian module is weakly Laskerian.

iii) Recall that a module $M$ is said to have finite Goldie dimension if $M$ does not contain an infinite direct sum of nonzero submodules, or equivalently, the injective envelope $\mathrm{E}(M)$ of $M$ decomposes as a finite direct sum of indecomposable injective submodules. Because for any $R$-module $C$, we have $\operatorname{Ass}_{R}(C)=\operatorname{Ass}_{R}(\mathrm{E}(C))$, it turns out that any module with finite Goldie dimension has only finitely many associated prime ideals. This yields that a module all of whose quotients have finite Goldie dimension is weakly Laskerian.

iv) Let $E$ be the minimal injective cogenerator of $R$ and $M$ an $R$-module. If for an $R$-module $M$ the natural map from $M$ to $\operatorname{Hom}_{R}\left(\operatorname{Hom}_{R}(M, E), E\right)$ is an isomorphism, then $M$ is said to be Matlis reflexive. By [1, Theorem 12], an $R$-module $M$ is Matlis reflexive if and only if $M$ has a finitely generated submodule $S$ such that $M / S$ is Artinian and $R / \operatorname{Ann}_{R} M$ is a complete semi-local ring. Also, as is mentioned in [5. Corollary 1.2], one can deduce from the argument 4, Proposition 1.3] that any quotient of an $R$-module $M$ has finite Goldie dimension if and only if $M$ has a finitely generated submodule $S$ such that $M / S$ is Artinian. Thus, by (iii), any Matlis reflexive module is weakly Laskerian.

v) An $R$-module $M$ is said to be linearly compact if each system of congruences

$$
x \equiv x_{i}\left(M_{i}\right),
$$

indexed by a set $I$ and where the $M_{i}$ are submodules of $M$, has a solution $x$ whenever it has a solution for every finite subsystem. It is known that the category of linearly compact $R$-modules forms a Serre subcategory of the category of all $R$-modules. In particular, every quotient of a linearly compact module is also linearly compact. 
On the other hand, a linearly compact module $M$ has finite Goldie dimension (see e.g. [13, Chapter 1.3]). Thus, if $M$ is a linearly compact module, then any quotient of $M$ has finite Goldie dimension, and so $M$ is weakly Laskerian by (iii).

To prove the main result of this paper, we need to prove the following two lemmas.

Lemma 2.3. i) Let $0 \longrightarrow L \longrightarrow M \longrightarrow N \longrightarrow 0$ be an exact sequence of $R$ modules. Then $M$ is weakly Laskerian if and only if $L$ and $N$ are both weakly Laskerian. Thus any subquotient of a weakly Laskerian module as well as any finite direct sum of weakly Laskerian modules is weakly Laskerian.

ii) Let $M$ and $N$ be two $R$-modules. If $M$ is weakly Laskerian and $N$ is finitely generated, then $\operatorname{Ext}_{R}^{i}(N, M)$ and $\operatorname{Tor}_{i}^{R}(N, M)$ are weakly Laskerian for all $i \geq 0$.

Proof. The proof of i) is easy and we leave it to the reader.

ii) We only prove the assertion for the Ext modules, and the proof for the Tor modules is similar. Because $R$ is a Noetherian ring and $N$ is finitely generated, it follows that $N$ possesses a free resolution

$$
F .: \ldots \longrightarrow F_{n} \stackrel{d_{n}}{\longrightarrow} F_{n-1} \stackrel{d_{n-1}}{\longrightarrow} \ldots \stackrel{d_{2}}{\longrightarrow} F_{1} \stackrel{d_{1}}{\longrightarrow} F_{0} \longrightarrow 0,
$$

consisting of finitely generated free modules. If $F_{i}=\bigoplus^{n} R$ for some integer $n$, then $\operatorname{Ext}_{R}^{i}(N, M)=H^{i}\left(\operatorname{Hom}_{R}(F, M)\right)$ is a subquotient of $\bigoplus^{n} M$. Therefore, it follows from (i), that $\operatorname{Ext}_{R}^{i}(N, M)$ is weakly Laskerian for all $i \geq 0$.

By [11, Theorem 11.38], there is a Grothendieck spectral sequence with $E_{2}^{p, q}:=$ $\operatorname{Ext}_{R}^{p}\left(R / \mathfrak{a}, H_{\mathfrak{a}}^{q}(M)\right) \underset{p}{\Longrightarrow} \operatorname{Ext}_{R}^{p+q}(R / \mathfrak{a}, M)$. Let $E_{\infty}:=\left\{E_{\infty}^{p, q}\right\}$ be the limit term of this spectral sequence. In the sequel, we show that if $M$ is weakly Laskerian, then $\operatorname{Ass}_{R}\left(E_{\infty}^{p, q}\right)$ is finite for all $p, q$ with $0 \leq p \leq q$.

Lemma 2.4. Let $\mathfrak{a}$ be an ideal of $R$. If $M$ is a weakly Laskerian module, then the set of associated primes of $E_{\infty}^{p, q}$ is finite for all $p, q$ with $0 \leq p \leq q$.

Proof. Since, by [11, Theorem 11.38], the Grothendieck spectral sequence $E_{2}^{p, q}=$ $\operatorname{Ext}_{R}^{p}\left(R / \mathfrak{a}, H_{\mathfrak{a}}^{q}(M)\right)$ converges to $H^{p+q}:=\operatorname{Ext}_{R}^{p+q}(R / \mathfrak{a}, M)$, it follows that there is a finite filtration

$$
0=\phi^{q+1} H^{q} \subseteq \phi^{q} H^{q} \subseteq \cdots \subseteq \phi^{1} H^{q} \subseteq \phi^{0} H^{q}=H^{q}
$$

of $H^{q}$ such that $E_{\infty}^{p, q} \cong \phi^{p} H^{q} / \phi^{p+1} H^{q}$ for all $p=0,1, \ldots, q$. Because $M$ is weakly Laskerian, by Lemma 2.3 (ii), it turns out that $\operatorname{Ext}_{R}^{q}(R / \mathfrak{a}, M)$ is also weakly Laskerian. Hence any subquotient of $H^{q}$ is weakly Laskerian. In particular, $\operatorname{Ass}_{R}\left(E_{\infty}^{p, q}\right)$ is finite.

Now, we are ready to prove the main theorem of this paper.

Theorem 2.5. Let $\mathfrak{a}$ be an ideal of $R$ and $M$ a weakly Laskerian $R$-module. Let $t$ be a natural integer. There is a finite subset $X$ of $\operatorname{Spec} R$ such that

$$
\operatorname{Ass}_{R}\left(\operatorname{Ext}_{R}^{l}\left(R / \mathfrak{a}, H_{\mathfrak{a}}^{t}(M)\right)\right) \subseteq\left(\bigcup_{0 \leq i<t, 0 \leq j \leq t^{2}+1} \operatorname{Ass}_{R}\left(\operatorname{Ext}_{R}^{j}\left(R / \mathfrak{a}, H_{\mathfrak{a}}^{i}(M)\right)\right)\right) \cup X,
$$

for $l=0,1$. 
Proof. Consider the Grothendieck spectral sequence

$$
E_{2}^{p, q}:=\operatorname{Ext}_{R}^{p}\left(R / \mathfrak{a}, H_{\mathfrak{a}}^{q}(M)\right) \underset{p}{\Longrightarrow} \operatorname{Ext}_{R}^{p+q}(R / \mathfrak{a}, M) .
$$

Set

$$
X=\left(\bigcup_{0 \leq j \leq t^{2}+1} \operatorname{Ass}_{R}\left(\operatorname{Ext}_{R}^{j}\left(R / \mathfrak{a}, H_{\mathfrak{a}}^{0}(M)\right)\right)\right) \cup \operatorname{Ass}_{R}\left(E_{\infty}^{0, t}\right) \cup \operatorname{Ass}_{R}\left(E_{\infty}^{1, t}\right) .
$$

Then $X$ is a finite set, by Lemmas 2.3 and 2.4. First, we prove the claim for $l=0$. We have to show that

$$
\operatorname{Ass}_{R}\left(E_{2}^{0, t}\right) \subseteq\left(\bigcup_{0 \leq i<t, 0 \leq j \leq t^{2}+1} \operatorname{Ass}_{R}\left(E_{2}^{j, i}\right)\right) \cup X .
$$

From the choice of $X$, it is clear that we may assume $M$ is $\mathfrak{a}$-torsion free. The exact sequence

$$
0 \longrightarrow \operatorname{ker} d_{i}^{0, t} \longrightarrow E_{i}^{0, t} \stackrel{d_{i}^{0, t}}{\longrightarrow} E_{i}^{i, t-i+1}
$$

yields that $\operatorname{Ass}_{R}\left(E_{i}^{0, t}\right) \subseteq \operatorname{Ass}_{R}\left(\operatorname{ker} d_{i}^{0, t}\right) \cup \operatorname{Ass}_{R}\left(E_{i}^{i, t-i+1}\right)$ for all $i \geq 2$. But ker $d_{i}^{0, t}=$ $E_{i+1}^{0, t}$, because $E_{i}^{-i, t+i-1}=0$. Hence

$$
\operatorname{Ass}_{R}\left(E_{i}^{0, t}\right) \subseteq \operatorname{Ass}_{R}\left(E_{i+1}^{0, t}\right) \cup \operatorname{Ass}_{R}\left(E_{i}^{i, t-i+1}\right),
$$

for all $i \geq 2$.

Let $n>t$ be an integer, and consider the sequence

$$
E_{n}^{-n, t+n-1} \stackrel{d_{n}^{-n, t+n-1}}{\longrightarrow} E_{n}^{0, t} \stackrel{d_{n}^{0, t}}{\longrightarrow} E_{n}^{n, t-n+1} .
$$

Since $M$ is a-torsion free, $E_{n}^{n, 0}=0$. Note that for each $i \geq 2$, the module $E_{i}^{p, q}$ is a subquotient of $E_{2}^{p, q}$. Also, $E_{n}^{i, j}=0$ if either $i<0$ or $j<0$. Thus, we have $\operatorname{ker} d_{n}^{0, t}=E_{n}^{0, t}$ and $\operatorname{im} d_{n}^{-n, t+n-1}=0$, and so

$$
E_{n+1}^{0, t}=\operatorname{ker} d_{n}^{0, t} / \operatorname{im} d_{n}^{-n, t+n-1} \cong E_{n}^{0, t} .
$$

Using (2) successively for all $n>t$, we get $E_{t+1}^{0, t} \cong E_{t+2}^{0, t} \cong \cdots=E_{\infty}^{0, t}$. Now, by iterating (1) for all $i=2, \ldots, t$, we deduce that

$$
\operatorname{Ass}_{R}\left(E_{2}^{0, t}\right) \subseteq\left(\bigcup_{i=2}^{t} \operatorname{Ass}_{R}\left(E_{i}^{i, t-i+1}\right)\right) \cup X .
$$

Next, we show that

$$
\operatorname{Ass}_{R}\left(E_{i}^{i, t-i+1}\right) \subseteq \bigcup_{k=1}^{t} \operatorname{Ass}_{R}\left(E_{2}^{k i, t-k i+k}\right),
$$

for all $i=3, \ldots, t$. Clearly this finishes the proof for the case $l=0$. Consider the exact sequence

$$
0 \longrightarrow \operatorname{ker} d_{i}^{k i, t-k i+k} \longrightarrow E_{i}^{k i, t-k i+k} \stackrel{d_{i}^{k i, t-k i+k}}{\longrightarrow} E_{i}^{(k+1) i, t-(k+1) i+k+1} .
$$

Since ker $d_{i}^{k i, t-k i+k} \subseteq \operatorname{ker} d_{2}^{k i, t-k i+k} \subseteq E_{2}^{k i, t-k i+k}$ and $E_{i}^{p, q}=0$ for all $q \leq 0$, by using the above exact sequence successively for $k=1,2, \ldots, t$, we deduce that $\operatorname{Ass}_{R}\left(E_{i}^{i, t-i+1}\right) \subseteq \bigcup_{k=1}^{t} \operatorname{Ass}_{R}\left(E_{2}^{k i, t-k i+k}\right)$. 
Now, by repeating the above argument, we can show that

$$
\operatorname{Ass}_{R}\left(E_{2}^{1, t}\right) \subseteq\left(\bigcup_{0 \leq i<t, 0 \leq j \leq t^{2}+1} \operatorname{Ass}_{R}\left(E_{2}^{j, i}\right)\right) \cup X .
$$

Therefore the proof is complete.

Now, we can obtain the following extension of [2, Theorem 2.2]. Note that, because $\operatorname{Supp}_{R}\left(H_{\mathfrak{a}}^{i}(M)\right) \subseteq \mathrm{V}(\mathfrak{a})$, it follows that $H_{\mathfrak{a}}^{i}(M)$ is $\mathfrak{a}$-cofinite whenever it is finitely generated.

Corollary 2.6. Let $\mathfrak{a}$ be an ideal of $R$ and $M$ a weakly Laskerian module. Let $t \in \mathbb{N}_{0}$ be an integer such that $H_{\mathfrak{a}}^{i}(M)$ is $\mathfrak{a}$-cofinite for all $i<t$. Then, the sets of associated primes of $H_{\mathfrak{a}}^{t}(M)$ and of $\operatorname{Ext}_{R}^{1}\left(R / \mathfrak{a}, H_{\mathfrak{a}}^{t}(M)\right)$ are finite.

Proof. If $t=0$, then the claim follows by Lemma 2.3. Now assume that $t>$ 0 , and let $i<t$ be an integer. Because $H_{\mathfrak{a}}^{i}(M)$ is a-cofinite for any $j \geq 0$, $\operatorname{Ext}_{R}^{j}\left(R / \mathfrak{a}, H_{\mathfrak{a}}^{i}(M)\right)$ is finitely generated, and so $\operatorname{Ass}_{R}\left(\operatorname{Ext}_{R}^{j}\left(R / \mathfrak{a}, H_{\mathfrak{a}}^{i}(M)\right)\right)$ is finite. On the other hand, since $\operatorname{Supp}_{R}\left(H_{\mathfrak{a}}^{t}(M)\right) \subseteq \mathrm{V}(\mathfrak{a})$, we have

$$
\operatorname{Ass}_{R}\left(\operatorname{Hom}_{R}\left(R / \mathfrak{a}, H_{\mathfrak{a}}^{t}(M)\right)\right)=\operatorname{Ass}_{R}\left(H_{\mathfrak{a}}^{t}(M)\right) \cap \mathrm{V}(\mathfrak{a})=\operatorname{Ass}_{R}\left(H_{\mathfrak{a}}^{t}(M)\right) .
$$

Therefore the conclusion follows by Theorem 2.5 .

It is clear by Theorem 2.5, that $\operatorname{Ass}_{R}\left(H_{\mathfrak{a}}^{t}(M)\right)$ is finite whenever the sets of associated primes of the modules $\operatorname{Ext}_{R}^{j}\left(R / \mathfrak{a}, H_{\mathfrak{a}}^{i}(M)\right)$ are finite for all $0 \leq i<t$ and all $0 \leq j \leq t^{2}+1$. Thus Lemma 2.3 (ii) yields the following.

Corollary 2.7. Let $\mathfrak{a}$ be an ideal of $R$ and $M$ a weakly Laskerian module. Let $t \in \mathbb{N}_{0}$ be an integer such that $H_{\mathfrak{a}}^{i}(M)$ is a weakly Laskerian module for all $i<t$. Then, the sets of associated primes of $H_{\mathfrak{a}}^{t}(M)$ and of $\operatorname{Ext}_{R}^{1}\left(R / \mathfrak{a}, H_{\mathfrak{a}}^{t}(M)\right)$ are finite.

Remark 2.8. Let $M$ be a finitely generated $R$-module. Khashyarmanesh and Salarian [9, Theorem $\mathrm{B}(\beta)$ ] have proved that if $t$ is an integer such that $\operatorname{Supp}_{R}\left(H_{\mathfrak{a}}^{i}(M)\right)$ is finite for all $i<t$, then the set of associated primes of $H_{\mathfrak{a}}^{t}(M)$ is finite. Clearly any $R$-module with finite support is weakly Laskerian. Hence Corollary 2.7 generalizes [9, Theorem $\mathrm{B}(\beta)]$.

\section{REFERENCES}

1. R. Belshoff, E. Enochs and J. R. Garcia Rozas, Generalized Matlis duality, Proc. Amer. Math. Soc., 128(5) (2000), 1307-1312. MR:1641645 (2000j:13015)

2. M. P. Brodmann and F. A. Lashgari, A finiteness result for associated primes of local cohomology modules, Proc. Amer. Math. Soc., 128(10) (2000), 2851-2853. MF.1664309 (2000m:13028)

3. M. P. Brodmann and R. Y. Sharp: 'Local cohomology-An algebraic introduction with geometric applications', Cambr. Univ. Press, 1998. MR1613627 (99h:13020)

4. E. Enochs, Flat covers and flat cotorsion modules, Proc. Amer. Math. Soc., 92(2) (1984), 179-184. MR.0754698 (85j:13016)

5. C. Faith and D. Herbera, Endomorphim rings and tensor products of linearly compact modules, Comm. Algebra, 25(4) (1997),1215-1255. MR1437670 (98b:16026)

6. R. Hartshorne, Affine duality and cofiniteness, Invent. Math., 9 (1970), 145-164. MR0257096 (41:1750)

7. C. Huneke, Problems on local cohomology, Free resolutions in commutative algebra and algebraic geometry, Res. Notes Math., 2 (1992), 93-108. MR1165320 (93f:13010)

8. K. I. Kawasaki, Cofiniteness of local cohomology modules for principle ideals, Bull. London Math. Soc., 30 (1998), 241-246. MR.1608094(98m:13025)

9. K. Khashyarmanesh and Sh. Salarian, On the associated primes of local cohomology modules, Comm. Algebra, 27(12) (1999), 6191-6198. MR1726302 (2000m:13029) 
10. G. Lyubeznik, A partial survey of local cohomology, Local cohomology and its applications, Lecture Notes in Pure and Appl. Math., 226 (2002), 121-154. MR.1888197 (2003b:14006)

11. J. Rotman, Introduction to homological algebra, Academic Press, $1979 . \quad$ MR0538169 (80k:18001)

12. A. K. Singh, p-torsion elements in local cohomology modules, Math. Res. Lett., 7(2-3) (2000), 165-176. MR1764314 (2001g:13039)

13. W. Xue, Rings with Morita duality, Lecture Notes in Mathematics, 1523, Springer-Verlag, Berlin, 1992. MR1184837 (94b:16002)

14. K. I. Yoshida, Cofiniteness of local cohomology modules for ideals of dimension one, Nagoya Math. J., 147 (1997), 179-191. MR1475172(98j:13014)

Department of Mathematics, Az-Zahra University, Vanak, Post Code 19834, Tehran, Iran - and - Institute for Studies in Theoretical Physics and Mathematics, P. O. Box 19395-5746, TEHRAN, IRAN

E-mail address: kdivaani@ipm.ir

Institute of Mathematics, University for Teacher Education, 599 Taleghani Avenue, TEHRAN 15614, IRAN 